University of Nebraska - Lincoln

DigitalCommons@University of Nebraska - Lincoln

Faculty Publications, Department of Psychology

Psychology, Department of

2008

\title{
Trauma exposure influences cue elicited affective responses among smokers with and without a history of major depression
}

Dennis E. McChargue

University of Nebraska-Lincoln, dmcchargue2@unl.edu

Alicia K. Klanecky

University of Nebraska-Lincoln, aliciaklanecky@creighton.edu

Kate Walsh

University of Nebraska-Lincoln, kate.walsh@einstein.yu.edu

David DiLillo

University of Nebraska-Lincoln, ddilillo@unl.edu

Follow this and additional works at: https://digitalcommons.unl.edu/psychfacpub

Part of the Psychiatry and Psychology Commons

McChargue, Dennis E.; Klanecky, Alicia K.; Walsh, Kate; and DiLillo, David, "Trauma exposure influences cue elicited affective responses among smokers with and without a history of major depression" (2008). Faculty Publications, Department of Psychology. 401.

https://digitalcommons.unl.edu/psychfacpub/401

This Article is brought to you for free and open access by the Psychology, Department of at DigitalCommons@University of Nebraska - Lincoln. It has been accepted for inclusion in Faculty Publications, Department of Psychology by an authorized administrator of DigitalCommons@University of Nebraska - Lincoln. 
Published in Addictive Behaviors 33:11 (November 2008), pp. 1454-1462; special issue on Anxiety, Stress and Trauma: Comorbidity with Tobacco and Marijuana Use and Disorders; doi: 10.1016/j.addbeh.2008.04.008 Copyright (c) 2008 Elsevier Ltd. Used by permission. http://www.elsevier.com/wps/product/cws home/471

Published online May 9, 2008.

\title{
Trauma exposure influences cue elicited affective responses among smokers with and without a history of major depression
}

\author{
Dennis E. McChargue, Alicia K. Klanecky, Kate Walsh, and David DiLillo \\ Department of Psychology, University of Nebraska-Lincoln, Lincoln NE 68588-0308 USA \\ Corresponding author - D. E. McChargue, 238 Burnett Hall, Department of Psychology, University of Nebraska- \\ Lincoln, Lincoln, NE 68588-0308 USA; tel 402 472-3197, fax 402 472-4637, email dmcchargue2@unl.edu
}

\begin{abstract}
The current study tested the emotional reactivity of smokers with and without histories of major depression (MDD Hx) and trauma exposure (TE). Four counterbalanced conditions nested negative (e.g., dysphoric) or neutral mood inductions with in vivo versus control smoking paraphernalia cues (Neutral+Control; Neutral+Cigarette; Neg+Control; Neg+Cigarette). Mixed model analysis of covariance (ANCOVA) tested between and within subjects differences in negative affective symptoms pre- to post-exposure across four groups (TE+MDD Hx; TE only; MDD Hx only; no history). Results produced two notable effects. First, TE only individuals endorsed the greatest increase in depressive symptoms across both negative mood induction conditions (regardless of smoking paraphernalia) compared with other groups. Second, dual history participants (TE+MDD Hx) show a potentiated depressive response to the Neg+Cigarette condition compared with the Neg+Control condition. Implications to a depression-specific negative affective vulnerability among TE only smokers that is independent of MDD Hx and greater than smokers with a MDD Hx are discussed.
\end{abstract}

Keywords: trauma, major depression, cigarette smoking, affect

\section{Introduction}

In most studies (Boscarino et al., 2002; Boyd et al., 1997; Breslau, 2002; Brown et al., 2000; Creamer et al., 2005; Galea et al., 2002; Shalev et al., 1998), but not all (Breslau, Davis, Peterson, \& Schultz, 2000), exposure to traumatic life events (TE) has been associated with the development of or the co-occurrence with severe psychopathology, such as Major Depressive Disorder histories (MDD Hx) and/or Posttraumatic Stress Disorder (PTSD). Such TE-related disorders also influence the onset of cigarette smoking behavior (e.g., Koenen et al., 2005; Koenen et al., 2006; Rauch et al., 2006) and are associated with greater smoking prevalence rates (e.g., Acierno et al., 1996; Acierno et al., 2000) compared with the general population (Centers for Disease Control, 2005). Despite this evidence, most research is focused on PTSD-related cigarette smoking (Feldner et al., 2007; Fu et al., 2007) with very little attention placed on other psychological sequelae, such as TE-related MDD Hx. Given that approximately 77\% of the TE population does not develop PTSD (Breslau, Davis, Andreski \& Peterson, 1991), broadening knowledge of health risk behavior among subgroups of non-PTSD smokers with a TE history (TE only) would provide important information for the study of trauma on smoking behavior.

Research suggests that lifetime violent assault status with a MDD Hx predicts $45 \%$ of current smokers compared with TE only (non-PTSD) individuals without a history of MDD (30\%) and non-vulnerable individuals (23\%; Acierno et al., 1996). TE only and MDD Hx also produce independent additive effects on smoking-related health risks (Benyamini and Solomon, 2005; Kramer et al., 2003; Links and Comstock, 1990; Spertus et al., 2003) and on health care utilization (Adams et al., 2006; Kates and Mach, 2007; Kramer et al., 2003). Little is unknown about biobehavioral mechanisms that may uniquely influence such dually vulnerable (TE + MDD Hx) compared with singularly vulnerable (e.g., MDD Hx without TE or TE only) and non-vulnerable smokers. 
One mechanism that may help differentiate dually vulnerable smokers from singularly vulnerable and non-vulnerable smokers is a selective susceptibility to negative affective states (i.e., increased vulnerability to experience negative affective states). Selective emotional responsiveness, particularly among subgroups of depression-prone smokers, may increase a person's drive to smoke to dispel these distressing emotional states (Spring et al., 2007). Most research exploring affective processing among TE only populations have shown that TE only veterans have greater negative affective reactions to trauma-related and non-trauma-related stressor scripts compared with neutral scripts (Beckham et al., 2007). After controlling for nicotine dependence and current MDD, trauma-related scripts compared with neutral scripts also were shown to shorten cigarette smoking puff onset intervals (McClernon et al., 2005). Lastly, TE only and MDD Hx individuals, independently, self-report smoking for negative affect reduction reasons (Beckham et al., 2004; Feldner et al., 2007; Feldner et al., 2007; Haaga et al., 2004; McChargue et al., 2004).

Despite suggestions that individuals with TE only or a MDD Hx may smoke for negative affective reasons (e.g., Feldner et al., 2007; Feldner et al., 2007; McChargue et al., 2004) and that, at least, TE only smokers show the expected negative affect reactivity to trauma-related stressors (e.g., Beckham et al., 2007), laboratory studies have yet to show that smokers with this dual vulnerability are disproportionately prone to negative affect compared with singularly vulnerable (i.e., those with TE only or MDD Hx only) and non-vulnerable smokers. Therefore, the purpose of the present study was to address gaps in extant literature by testing for interactive effects of TE only and MDD Hx on affective reactivity among smokers. Our general linear hypothesis is that negative affective elevations resulting from non-trauma-related stressors compared with neutral emotional stimuli would be the greatest in the dually vulnerable group followed, respectively, by the singularly vulnerable and non-vulnerable groups. Given that environmental smoking cues may increase negative affective substrates associated with use (e.g., Doran et al., in press; Drobes et al., 2006), emotional manipulations (stressor vs. neutral) were stratified across in vivo cigarette cue and neutral environmental cue exposure to explore for possible potentiated negative affect reactivity that results from the combination of negative mood induction with the presence of an environmental smoking cue. This was done because, for some, environmental smoking cues appear to be needed to selectively prime internal drives (negative mood) associated with use during stressful situations (Doran et al., in press).

\section{Method}

\subsection{Participants}

The current study was a secondary analysis of a larger study featuring smokers with and without a MDD Hx. Given the high comorbidity with this population and those with a history of trauma (Brady et al., 2000; Maes et al., 2000; Pfefferbaum et al., 2002), it was deemed a suitable sample in which to assess the study question after controlling for nicotine and depression-related variables. Participant recruitment was accomplished by distributing flyers and newspaper advertisements in a large Midwestern city. Ineligible persons included those with current mental health disorders other than nicotine dependence, those actively using smoking cessation techniques, those abstinent less than 6 months from a previous substance dependency other than nicotine, those unable to read the questionnaires, those on psychiatric medications (other than antidepressant medication that was stabilized for 2 months), and those younger than 21 or older than 55. Additionally, the ethnic representation of the sample reflected the surrounding community and participant selection was stratified by gender. Smoking severity as measured by the FTND (See Section 2.3.4) indicated that $6.3 \%$ of participants reported very low nicotine dependence, $19.0 \%$ low dependence, $16.5 \%$ medium dependence, $38.0 \%$ high dependence, and $20.2 \%$ very high dependence. For univariate statistics see Table 1.

\subsection{Participant flow}

Initially, 274 participants responded to the advertisements and were screened via telephone. Seventy-two individuals (26.2\%) were found ineligible during the telephone screening for the following reasons: $38 \%$ currently psychiatrically medicated, $25 \%$ uninterested in participating, $14 \%$ not meeting stratification requirements, $5 \%$ high blood pressure, and $5 \%$ currently in treatment with the remaining individuals found ineligible due to current drug use, smoking less than what was required by the study ( $<15$ cigarettes per day), recent cessation attempts, age requirements, disconnected telephone, and pregnancy, each contributing less than 3\%. After telephone screening completion, 202 individuals were deemed initially eligible and scheduled for the screening visit; $96(48 \%)$ did not attend. Accordingly, 106 participants consented to the study with an additional 27 (25\%) not completing for the following reasons: 17 (63\%) did not complete experimental sessions, three $(11 \%)$ met criteria for current Axis I disorders, three $(11 \%)$ attempted to participate in the study more than once after being found ineligible, two $(7 \%)$ did not report exclusionary medication use until screening session, one person (4\%) was referred to a physician due to a high carbon monoxide reading, and one person $(4 \%)$ was dropped for missing more than three scheduled sessions. In total, 79 participants completed the study. 
Table 1. Summary of univariate and bivariate statistics

\begin{tabular}{|c|c|c|}
\hline \multirow{2}{*}{$\frac{\text { Variable }}{\text { Age }}$} & \multicolumn{2}{|c|}{ Univariate statistics $M(\mathrm{~S})$ or frequency (\%) } \\
\hline & 40.85 & $(10.59)$ \\
\hline \multicolumn{3}{|l|}{ Ethnicity } \\
\hline African American & 51 & $(68.0 \%)$ \\
\hline Caucasian & 24 & $(32.0 \%)$ \\
\hline \multicolumn{3}{|l|}{ Gender } \\
\hline Male & 44 & $(55.7 \%)$ \\
\hline Female & 35 & $(44.3 \%)$ \\
\hline \multicolumn{3}{|l|}{ Trauma } \\
\hline $\mathrm{TE}$ & 26 & $(32.9 \%)$ \\
\hline \multicolumn{3}{|l|}{ History of MDD } \\
\hline Yes & 40 & $(50.6 \%)$ \\
\hline Number of prior MDD episodes & 1.29 & $(2.05)$ \\
\hline FTND total & 5.86 & $(2.08)$ \\
\hline DPI total & 30.33 & (11.73) \\
\hline DAS total & 172.74 & $(54.85)$ \\
\hline \multicolumn{3}{|l|}{ Anhedonia $^{\mathrm{a}}$} \\
\hline TE & 118.69 & (11.39) \\
\hline No TE & 121.51 & $(16.25)$ \\
\hline \multicolumn{3}{|l|}{ Anger ${ }^{\mathrm{a}}$} \\
\hline TE & 6.21 & $(7.86)$ \\
\hline No TE & 6.08 & $(8.64)$ \\
\hline \multicolumn{3}{|l|}{ Anxiety ${ }^{a}$} \\
\hline TE & 8.63 & $(5.71)$ \\
\hline No TE & 7.57 & $(6.70)$ \\
\hline
\end{tabular}

$N=79 ;$ a $=$ bivariate statistical analyses.

\subsection{Measures}

\subsubsection{Caffeine and alcohol intake}

Given evidence that suggests daily consumption of alcohol and caffeine may influence cue reactivity (Cooney et al., 1997; Smith et al., 2003), a beverage score was assessed. As with our prior studies (McChargue \& Doran, 2009), the beverage intake form comprised 6 questions that assessed the amount of coffee, tea, soda, spirits, wine and beer that was consumed within the last $24 \mathrm{~h}$ prior to the exposure session. A total beverage consumption score was derived by summing the number from each question. Scores ranged from 0 to 20.

\subsubsection{Depression Proneness Inventory}

Demonstrating bivariate relations with TE and MDD Hx, the Depression Proneness Inventory (DPI) measures risk to depressive symptomatology including cognitive vulnerabilities, tendencies to feel inadequate and experience depressive symptoms, and recent experiences of depressive symptoms. Showing good convergent validity, the DPI is a strong predictor of depression history and affective risk factors for future depression proneness (Strong, Brown, Kahler, Lloyd-Richardson, \& Niaura, 2004). Cronbach's alpha equaled 92.

2.3.3. Dysfunctional Attitude Scale

Designed to assess cognitive thinking errors associated with depression, the Dysfunctional Attitude Scale (DAS; Beck, Steer, Brown, \& Weissman, 1991) is a 40-item scale comprised of two factors: a factor measuring attitudes about achievement and a factor associated with attachment and interpersonal relationships. Incorporated as a covariate to provide a cognitive measure associated with a MDD Hx, the DAS has shown good test-retest reliability (.73), convergent validity (Oliver \& Baumgart, 1985), and internal consistency (Cronbach's alpha $=.93$ in the current study).

\subsubsection{Fagerstrom Test for Nicotine Dependence}

Revised from the Fagerstrom Tolerance Questionnaire, the Fagerstrom Test for Nicotine Dependence (FTND; Heatherton, Kozlowski, Frecker, \& Fagerstrom, 1991) is a widely used measure of nicotine dependence and operated as a covariate in the current study to control for participant smoking levels. The brief, 6-item questionnaire produces scores ranging from 0-10 and uses categorical descriptions to interpret an individual's dependence severity. The FTND has shown high test-retest reliability and convergent validity (Buckley et al., 2005; Fagerstrom and Schneider, 1989), positively related to smokers' baseline carbon monoxide levels $(r=.33)$ and cigarettes smoked per day $(r=.44)$ in the current study. Cronbach's alpha for the current study was .45 .

\subsubsection{The Fawcett-Clark Anhedonia Scale}

The Fawcett-Clark Anhedonia Scale (Fawcett, Clark, Scheftner, \& Gibbons, 1983) asks participants to rate current hedonic reactions to hypothetical pleasurable situations in a 36-item questionnaire. The instrument provided a measure of anhedonia included in the analyses to rule out subsyndromal PTSD symptoms (Kashdan, Elhai, \& Frueh, 2006). 
The scale items have been shown to effectively tap a single latent dimension (loss of pleasure) suggesting adequate internal consistency (Cronbach's alpha $=.93$ in current study) and have demonstrated good overall psychometric properties in clinical and non-clinical samples (Fawcett et al., 1983).

\subsubsection{Profile of Mood States}

The Profile of Mood States (POMS; McNair, Lorr, \& Droppelman, 1971) is a self-report questionnaire designed to measure transient affective states. Consisting of 65 adjectives rated on a 5-point Likert scale, the POMS subscales were utilized to measure changes in affective symptoms pre- to post-experimental conditions (independent variables). Additionally, selected subscales (i.e. anxiety, anger, and depression) provided baseline ratings of affective indicators used to rule out subsyndromal PTSD symptoms. The measure has shown good convergent and discriminant validity (Nyenhuis, Yamamoto, Luchetta, Terrien, \& Parmentier, 1999). Internal consistency for the POMS was measured across all four experimental sessions and Cronbach's alpha ranged from .96 to 97.

\subsubsection{Structured Clinical Interview for DSM-IV}

To determine study eligibility and independent variables, participants were screened using the Structured Clinical Interview for DSM-IV Non-Patient version (SCID-NP; First, Spitzer, Gibbon, \& Williams, 1996). The SCID-NP was specifically used to assess both the history and presence of mood disorders including major depressive disorder and number of previous episodes. Trauma exposure was also determined and defined as meeting criterion A for PTSD (i.e., the individual had experienced actual or threatened death or serious injury and responded with intense fear, helplessness, or horror). Individuals currently meeting full criteria or a lifetime diagnosis of PTSD were excluded from the study. The SCID-NP has been reported to have good-to-excellent validity as well as high reliability for most Axis I and Axis II disorders (Segal, Hersen, \& Van Hasselt, 1994).

\subsection{Procedure}

\subsubsection{Screening session}

Initially, assenting participants were screened over the telephone to evaluate demographic and general medical information; those meeting preliminary study criteria were scheduled for a screening visit. After obtaining written consent from all individuals, the current study's screening session assessed eligibility via clinical interview by the principle investigator (DEM) and a trained post-doctoral staff member using the SCID-NP. Weekly meetings were held to maintain clinical interview consensus. Next, an ecolyzer test was administered to assess smoking status via expired carbon monoxide samples and those still remaining eligible for the study (CO readings > 10) completed basal mood and smoking questionnaires. Guided imagery scripts utilized in the mood induction procedure were then generated (full description in Section 2.4.2.1).

\subsubsection{Experimental sessions}

Following the screening session, a counterbalanced series of four experimental sessions nested mood induction (negative vs. neutral) with environmental cue (in vivo cigarette vs. control cue). Participants were tested individually, and on testing days were asked to abstain from caffeine 0 prior to experimental session. No individual was scheduled before 11:00 am to reduce the impact of diurnal variations in mood. Further, ecolyzer readings and self-reported 24-h alcohol/ caffeine intake were measured at the start of each session. All participants then smoked one cigarette to prevent nicotine withdrawal and to standardize the time from their last cigarette. Following the cigarette, individuals rested for $30 \mathrm{~min}$ in a comfortable chair to stabilize mood effects. Baseline mood was recorded via self-report using the POMS questionnaire subscales (i.e. anxiety, depression, fatigue, vigor, confusion, and anger). After baseline assessments, mood + cue exposure procedures were simultaneously implemented for $10 \mathrm{~min}$ as described in Sections 2.4.2.1 and 2.4.2.2. Subsequent mood ratings were measured post-exposure via the POMS to assess the level of change from pre- to post-manipulation. Used to ensure adequate engagement in memory recall and response to the induction technique, memory vividness was rated on a 100-point scale at the 5 min mark of the mood induction (Tiffany \& Hakenewerth, 1991).

2.4.2.1. Mood induction procedure. Mood induction scripts were initially generated during the screening procedure. Participants were invited to verbally describe memories of four events in the past year which had caused feelings of "upset, very anxious, angry, or sad," as well as four events which did not elicit feelings of "upset or happy" (Litt, Cooney, Kadden, \& Gaupp, 1990). Next, participants used a 10-point Likert scale to indicate the degree to which each event made them feel sad, angry, or anxious. Events scoring a 7 or greater were scripted for the negative mood induction while those scoring a 0 or 1 were included in the neutral mood induction. During the experimental sessions, research assistants prompted participants to recall these previously generated negative memories. Further, participants undergoing the negative mood induction received headphones and listened to audiotaped pieces of classical music including Russia Under the Mongolian Yoke and Adagio Pour Cordes which have both been shown to induce negative mood (Clark and Teasdale, 1985; Gerrards-Hesse et al., 1994; Marin, 1990). In contrast, because prior research has shown music can evoke negative and positive mood states (Clark, 1983; Vaestfjaell, 2002), the neutral mood induction did not include a musical component. Instead, participants were prompted by research assistants to recall the previously rated neutral memory (e.g., doing laundry). All memory prompts given by research assistants were scripted and individuals who had completed the negative mood induction received a positive mood induction before leaving the 
laboratory to dispel any lingering negative feelings. Research has found this autobiographical technique successful in manipulating mood (Ekman, Levenson, \& Friesen, 1983).

2.4.2.2. Cue exposure procedure. When an in vivo cigarette cue was coupled with the mood induction procedure, participants were shown their brand of cigarettes, a lighter, and an ashtray. Prior to mood evocation, participants were instructed to light one cigarette (without putting the cigarette in their mouth) and hold the cigarette comfortably in their dominant hand until the research assistant asked them to extinguish it. In comparison, during the cigarette control condition, participants were shown a roll of scotch tape and instructed to hold the scotch tape in their dominant hand until the research assistant asked them to place it back on the tray.

\subsection{Analytic plan}

The current analysis was conducted using a mixed-group factorial analysis of covariance (ANCOVA) to examine the influence of trauma exposure (TE) and a history of major depressive disorder (MDD Hx) on differences in affective symptoms pre- to post-four Latin-squared counterbalanced experimental conditions (Tabachnick \& Fidell, 2001). Group was the primary between-subjects factor (TE+MDD Hx; TE only; MDD Hx only; no history). The within subjects factor was experimental condition that nested negative or neutral mood inductions with in vivo cigarette versus control environmental cues (Neutral+Control; Neutral+Cigarette; Neg+Control; Neg+Cigarette). The dependent variables reflected affective change scores (anger, anxiety, depression and vigor) from pre- to post-exposure. Greenhouse-Geisser estimates were used to adjust for any sphericity issues that were associated with multiple comparisons (Tabachnick \& Fidell, 2001).

Covariates (italicized) were chosen for statistical and theoretical purposes, based on the likelihood that they may influence the current results. Both TE and MDD Hx were significantly correlated with the number of prior major depressive episodes ( $r=.40$ and $r=.64$, respectively) and total score on the DPI $(r=.35$ and .48 , respectively) with all $p$ s $<.01$. Further, depression change scores in the neutral cig condition $(r=.23, p<.05)$ as well as the negative no cig condition $(r=-.29, p<.01)$ were significantly correlated with age while scores in the neutral no cig condition were related to ethnicity $(r=-.24, p<.05)$. Because study participants were smokers, total FTND scores were controlled to address severity of nicotine dependence. Also, because MDD symptom presentation differs across men and women (Khan, Gardner, Prescott, \& Kendler, 2002), gender was controlled. Lastly, prior research has reported a link between a history of MDD and elevated dysfunctional attitudes independent of subsyndromal depression symptoms (Otto et al., 2007). Consequently, DAS responses were included as a covariate to provide a cognitive descriptor of depressive characteristics. Antidepressant medication status, Latin-squared order of exposures and 24-h alcohol/caffeine intake were dropped from the analysis for lack of covariance with independent and dependent variables.

\section{Results}

\subsection{Preliminary analysis}

Initially, univariate statistics from individuals who completed the study versus individuals who were originally found eligible before the telephone screening and subsequent screening session were compared. Independent samples $t$-tests revealed no differences between demographic variables including age, ethnicity, gender ( $p s>.12)$ as well as smokingrelated variables including FTND and cigarettes smoked per day ( $p$ s > .36). Additionally, while no significant differences were found in depression-related variables $(p s>.62)$, those completing the study $(M=.81, \mathrm{SD}=.40)$ endorsed higher rates of trauma $[t(82)=-2.44, p=.02]$ compared to individuals who did not complete $(M=.56, \mathrm{SD}=.50)$.

To rule out the possibility that subsyndromal PTSD symptoms among the TE groups may influence results, screening session responses on several POMS indices were compared using one-way ANOVAs across individuals with and without TE. No group differences were found in screening session ratings of depression $[F(1,77)=3.19, p=.078$, Mse $=$ $58.15, r=.20]$, anhedonia $[F(1,77)=.63, p=.43$, Mse $=220.35, r=.09]$, anger $[F(1,73)=.004, p=.95$, Mse $=70.57, r=.007]$, or anxiety $[F(1,73)=.44, p=.51$, Mse $=41.04, r=.08]$. These findings are consistent with previous research reporting elevated symptoms of depression, anger, and anxiety scores among PTSD individuals compared to TE and no-trauma controls who reported no mean differences (Butler, Mueser, Sprock, \& Braff, 1996). See Table 1 for the bivariate means.

\subsection{Mood manipulation check}

Indicating successful participant response to the mood induction technique regardless of group, paired sample $t$-test demonstrated significant increases in negative affect pre- to post mood induction for both the Neg+Control condition $[t(77)=-8.08, p<.001, r=.68]$ and the Neg+Cigarette condition $[t(77)=-8.00, p<.001, r=.67]$. Negative affect did not significantly increase pre- to post neutral mood induction for either the cigarette $(p=.193)$ or environmental control condition $(p=.749)$. Overall, vividness ratings used to evaluate degree of memory engagement exceeded the third quartile in the Neutral+Control condition $(M=77.00, S D=25.10)$, Neutral+Cigarette condition $(M=79.29$, $\mathrm{SD}=22.73)$, Neg+Control condition $(M=76.22, \mathrm{SD}=25.43)$, and Neg+Cigarette condition $(M=78.62, \mathrm{SD}=22.14)$. Vividness results were comparable to previous studies (Tiffany and Drobes, 1990; Tiffany and Hakenewerth, 1991) while effectiveness of the negative mood induction procedure exceeded similar studies $(r=.53$ compared to the current $r s=.68$ and .67; Hufford, 2001). 


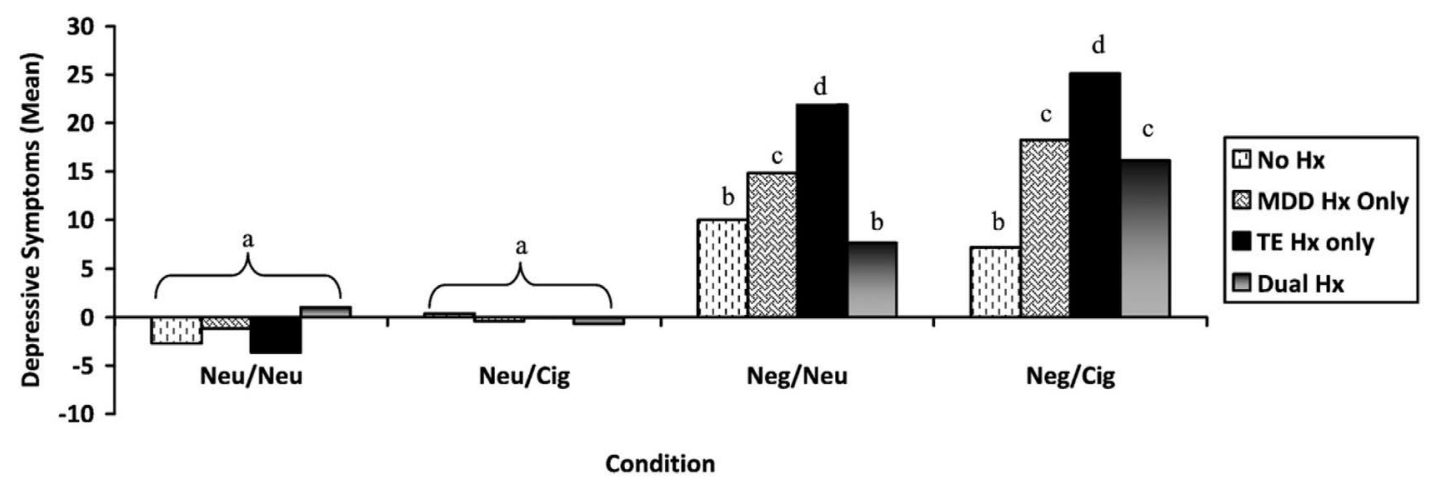

Figure 1. Summary of cell means for changes in depressive symptoms. Trauma Exposure (TE); History of Major Depressive Disor$\operatorname{der}(\mathrm{MDD} H \mathrm{Hx}) ; \mathrm{a}, \mathrm{b}, \mathrm{c}$, and d denote significant changes in depressive symptoms across group and experimental conditions (LSD mmd = 5.62); No Hx $n N=31$, Dual Hx $n N=18$, MDD Hx only $n N=17$, TE only $n N=41$; Neu = Neutral/control conditioning; Neg $=$ Negative $\operatorname{mood}$ induction; $\mathrm{Cig}=$ In vivo cigarette exposure.

\subsection{Primary analysis}

After controlling for noted covariates, ANCOVA analyses indicated that changes in anxiety, anger and vigor were statistically equivalent across groups during the four experimental conditions ( $p s>.05)$. Results also revealed a significant 3-way interaction among TE, MDD Hx and condition when examining participants' change in self-reported depression pre- to post-condition, $[F(3,177)=5.28, p=.006$, Mse $=70.51, r=.29]$. Cell means, LSD minimum mean difference and group sizes for the 3-way interaction are displayed in Figure 1. Significant group differences were found within the negative mood induction conditions and not the neutral condition. TE only individuals endorsed the greatest elevation in self-reported depression across both negative mood conditions (regardless of smoking paraphernalia) compared with other groups. MDD Hx only smokers showed similar effects, but to a lesser degree of TE only smokers. Further, dual history participants reported a dramatic increase in self-reported depression during the Neg+Cigarette condition compared with Neg+Control condition.

Results also showed a significant 2-way interaction between TE and MDD Hx $[F(1,59)=6.19, p=.02$, Mse $=114.40$, $r=.31$. Examination of simple effects indicated that TE only individuals reported a significantly greater change in self-reported depression compared to individuals in the no history group (no TE or MDD Hx). Lastly, there was a main effect of condition $[F(3,177)=9.54, p=.001$, Mse $=70.51, r=.37]$. Individuals reported a significantly greater change in self-reported depression during the negative mood conditions compared to the neutral mood conditions (regardless of smoking paraphernalia).

To exclude the possibility that spurious trauma-related scripts influenced the changes in depression across experimental conditions, negative mood induction scripts were evaluated for traumatic content and responses of TE individuals were assessed. While five (19.2\%) TE individuals received a negative mood script containing traumatic content; individuals who received trauma scripts were not disproportionately reactive compared with those who did not receive a trauma script. This suggests that the TE group findings were not influenced by depressive reports related to the trauma scripts.

\section{Discussion}

The overall study results produced expected and unexpected effects. Most notably, individuals with TE only histories showed the greatest elevations in negative mood-induced depressive symptoms compared with all other groups (i.e., non-vulnerable, MDD Hx only, dually vulnerable). This global depression reaction was also independent of cigarette cues. The MDD Hx group had similar global changes in depressive symptoms following both negative mood induction conditions, but to a lesser magnitude. Dually vulnerable smokers compared with non-vulnerable smokers showed significant depressive symptoms changes only during the negative mood induction plus in vivo cigarette condition. Lastly, null group effects were documented across the neutral mood induction conditions.

Research has examined emotional reactivity using negative affect as the manipulated emotion for TE only smokers (e.g., Beckham et al., 2007; McClernon et al., 2005), but has yet to examine negative affect-specific effects. This study is the first to implicate a depressive symptom vulnerability for non-trauma-related stressors that appears independent of a clinically significant MDD Hx and independent of other affective reactions (e.g., anxiety, anger, and vigor). Findings also suggest that negative affective smoking among TE only individuals may be specific to the alleviation of depression, thus suggesting the need to supplement smoking treatment for TE only smokers with psychosocial and/or pharmacological treatments for depression.

Contrary to expectations, dual history smokers reported fewer changes in depressive symptoms following the negative mood induction procedure compared to singularly vulnerable smokers (MDD only or TE only). Our finding may reflect a blunted affective response to the negative mood induction procedure such that dually vulnerable smokers had limited capacity to fully experience negative emotions, which is consistent with literature suggesting that 
numbing symptoms are a core trauma symptom (Litz \& Gray, 2002); however, future research is needed to more fully examine links among trauma exposure, a history of MDD, and depressive states.

The only exception to the finding of diminished negative emotional reactivity among dual history smokers was in the negative mood + in vivo cigarette condition. Dually vulnerable smokers showed self-reported depression elevations that were comparable to MDD Hx only smokers. These self-reported depression reactions were significantly greater than the reactions in the negative mood + environmental neutral cue and significantly greater than non-vulnerable smokers' reactions in both negative mood induction conditions (see Figure 1). As such, smokers with TE+MDD Hx showed a potentiated depressogenic effect when the smoking cue was paired with the negative mood induction, suggesting that smoking cues may eradicate the previously observed numbing response. These data may suggest that dually vulnerable smokers compared with non-vulnerable smokers are selectively susceptible to negative affective smoking only when smoking paraphernalia are present.

Our results are tempered by a few study limitations. First, cell sample sizes for each group were not equally stratified and the TE only group was substantially smaller in comparison. As a result, it may be important for future studies to replicate and extend these findings with equally stratified samples of vulnerable smokers. Second, it is possible that subsyndromal symptoms of PTSD or depression may have accounted for the increase in emotional reactivity observed among TE only individuals. To assuage such concerns, preliminary data show that groups did not differ at the screening session across subsyndromal states of anhedonia, anger, anxiety and depression. Third, a total of five people who reported TE were inadvertently presented with trauma-related scripts, making it possible that TE individuals evidenced a selectively heightened emotional response to the negative mood induction procedure because the mood induction scripts involved traumatic events rather than non-trauma-related stressors. Despite this possibility, comparison of script effects on affective changes showed that TE smokers who received trauma scripts did not demonstrate significantly different affective responses when compared to those receiving non-trauma-related stressor scripts. Because the primary dependent variable is self-reported affective change, it is also plausible that demand characteristics influenced the results. Concerns about potential participant demand characteristics were, however, minimized with the depression-specific findings. Given that the negative mood induction procedures were structured to illicit general negative affect, the lack of a global negative affective response across anxiety, anger, and depression ratings suggests that participants' self-reported affect may not have been unduly influence by social desirability. Finally, exclusionary criteria helped maintain the internal validity of our findings but diminished our ability to completely generalize the results.

In conclusion, the present study adds to extant literature that reports a potential negative affective mechanism linking non-PTSD-related TE with cigarette smoking (Beckham et al., 2007; Feldner et al., 2007; Feldner et al., 2007; McClernon et al., 2005) by showing that TE only smokers' affective vulnerability may be specific to depressive states, independent of MDD Hx, and greater than MDD Hx smokers' depressive vulnerability. We further show that dually vulnerable smokers are less reactive to stressors, but may be influenced by self-reported depressive changes when environmental smoking cues are present. Future studies should replicate these findings and extend them to examine self-reported depression changes during smoking abstinence. For example, future data showing that the present study's depressive vulnerability reactions extend to withdrawal-related depression that moderates relapse would further our understanding of affect-specific influences on smoking maintenance and relapse among TE only smokers.

\section{Acknowledgment}

This project was partially supported by NIH (DA00467) and VA Merit Review Entry Program grants to Dr. McChargue.

\section{References}

Acierno et al., $2000 \triangleright$ R. Acierno, D. G. Kilpatrick, H. Resnick, B. Saunders, M. D. Arellano, and C. Best, Assault, PTSD, family substance use, and depression as risk factors for cigarette use in youth: Findings from the national survey of adolescents, Journal of Traumatic Stress 13 (3) (2000), pp. 381-396.

Acierno et al., $1996>$ R. Acierno, D. G. Kilpatrick, H. S. Resnick, B. E. Saunders, and C. L. Best, Violent assault, posttraumatic stress disorder, and depression: Risk factors for cigarette use among adult women, Behavior Modification 20 (1996), pp. 363384.

Adams et al., $2006-$ R. E. Adams, J. A. Boscarino, and S. Galea, Social and psychological resources and health outcomes after the world trade center disaster, Social Science and Medicine 62 (1) (2006), pp. 176-188.

Beck et al., 1991 A. T. Beck, R. A. Steer, G. K. Brown, and A. Weissman, Factor analysis of the Dysfunctional Attitude Scale in a clinical population, Psychological Assessment 3 (1991), pp. 478-483.

Beckham et al., 2007 J. C. Beckham, M. F. Dennis, F. J. McClernon, S. L. Mozley, C. F. Collie, and S. R. Vrana, The effects of cigarette smoking on script-driven imagery in smokers with and without posttraumatic stress disorder, Addictive Behaviors 32 (2007), pp. 2900-2915.

Beckham et al., 2004 J. C. Beckham, P. R. Gehrman, F. J. McClernon, C. F. Collie, and M. E. Feldman, Cigarette smoking, ambulatory cardiovascular monitoring, and mood in Vietnam veterans with and without chronic posttraumatic stress disorder, Addictive Behaviors 29 (2004), pp. 1579-1593. 
Benyamini and Solomon, $2005 \triangleright$ Y. Benyamini and Z. Solomon, Combat stress reactions, posttraumatic stress disorder, cumulative life stress, and physical health among Israeli veterans twenty years after exposure to combat, Social Science $\mathcal{E}$ Medicine 61 (2005), pp. 1267-1277.

Boscarino et al., 2002 J. A. Boscarino, S. Galea, J. Ahern, H. Resnick, and D. Vlahov, Health services \#66 access to psychiatric services in New York city following the September 11th terrorist attacks, Annals of Epidemiology 12 (7) (2002), p. 514.

Boyd et al., 1997 C. Boyd, D. Henderson, P. Ross-Durow, and J. Aspen, Sexual trauma and depression in African-American women who smoke crack cocaine, Substance Abuse 18 (1997), pp. 133-141.

Brady et al., $2000 \triangleright$ K. T. Brady, T. K. Killeen, T. Brewerton, and S. Lucerini, Comorbidity of psychiatric disorders and posttraumatic stress disorder, Journal of Clinical Psychiatry 61 (2000), pp. 22-32.

Breslau, 2002 N. Breslau, Epidemiologic studies of trauma, posttraumatic stress disorder, and other psychiatric disorders, Canadian Journal of Psychiatry 47 (2002), pp. 923-929.

Breslau et al., $1991-$ N. Breslau, G. C. Davis, P. Andreski, and E. Peterson, Traumatic events and postraumatic stress disorder in an urban population of young adults, Archives of General Psychiatry 48 (1991), pp. 216-222.

Breslau et al., 2000 N. Breslau, G. C. Davis, E. L. Peterson, and L. R. Schultz, A second look at comorbidity in victims of trauma: The posttraumatic stress disorder-major depression connection, Biological Psychiatry 49 (9) (2000), pp. 902-909.

Brown et al., 2000 E. S. Brown, M. K. Fulton, A. Wilkeson, and F. Petty, The psychiatric sequelae of civilian trauma, Comprehensive Psychiatry 41 (2000), pp. 19-23.

Buckley et al., 2005 T. C. Buckley, S. L. Mozley, D. R. Holohan, K. Walsh, J. C. Beckham, and J. D. Kassel, A psychometric evaluation of the Fagerström Test for Nicotine Dependence in PTSD smokers, Addictive Behaviors 30 (5) (2005), pp. $1029-1033$.

Butler et al., 1996 R. W. Butler, K. T. Mueser, J. Sprock, and D. L. Braff, Positive symptoms of psychosis in Posttraumatic Stress Disorder, Biological Psychiatry 39 (1996), pp. 839-844.

Centers for Disease Control, 2005 Centers for Disease Control, Cigarette smoking among adults - United States, 2004, Morbidity \& Mortality Weekly Report 54 (2005), pp. 1121-1124.

Clark, 1983 D. M. Clark, On the induction of depressed mood in the laboratory: Evaluation and comparison of the Velten and musical procedures, Addictive Behavioral Research and Therapy 5 (1983), pp. 27-49.

Clark and Teasdale, 1985 D. M. Clark and J. D. Teasdale, Constraints on the effects of mood on memory, Journal of Personality and Social Psychology 48 (1985), pp. 1595-1608.

Creamer et al., $2005-$ M. Creamer, A. C. McFarlane, and P. Burgess, Psychopathology following trauma: The role of subjective experience, Journal of Affective Disorders 86 (2005), pp. 175-182.

Cooney et al., 1997 N. L. Cooney, M. D. Litt, P. A. Morse, L. O. Bauer, and L. Gaupp, Alcohol cue reactivity, negative-mood reactivity, and relapse in treated alcoholic men, Journal of Abnormal Psychology 106 (1997), pp. 243-250.

Doran et al., in press N. Doran, J. W. Cook, D. McChargue, M. Meyers, and B. Spring, Cue-elicited negative affect in impulsive smokers. Psychology of Addictive Behaviors (in press).

Drobes et al., 2006 D. J. Drobes, A. Elibero, and D. E. Evans, Attentional bias for smoking and affective stimuli: A Stroop task study, Psychology of Addictive Behaviors 20 (2006), pp. 490-495.

Ekman et al., $1983-$ P. Ekman, R. W. Levenson, and W. V. Friesen, Autonomic nervous system activity distinguishes among emotions, Science 221 (1983), pp. 1208-1210.

Fagerstrom and Schneider, 1989 K. O. Fagerstrom and N. G. Schneider, Measuring nicotine dependence: A review of the Fagerstrom Tolerance, Journal of Behavioral Medicine 12 (2) (1989), pp. 159-182.

Fawcett et al., $1983-$ J. Fawcett, D. C. Clark, W. A. Scheftner, and R. D. Gibbons, Assessing anhedonia in psychiatric patients: The pleasure scale, Archives of General Psychiatry 40 (1983), pp. 79-84.

Feldner et al., 2007 M. T. Feldner, K. A. Babson, and M. J. Zvolensky, Smoking, traumatic event exposure, and post-traumatic stress: A critical review of the empirical literature, Clinical Psychology Review 27 (2007), pp. 14-45.

Feldner et al., 2007 M. T. Feldner, K. A. Babson, M. J. Zvolensky, A. A. Vujanovic, S. F. Lewis, and L. E. Gibson et al., Posttraumatic stress symptoms and smoking to reduce negative affect: An investigation of trauma-exposed daily smokers, Addictive Behaviors 32 (2007), pp. 214-227.

First et al., 1996 M. B. First, R. L. Spitzer, M. Gibbon, and J. B. W. Williams, Structured clinical interview for DSM-IV Axis I Disorders Non-Patient Edition, Biometrics Research Department, New York (1996).

Fu et al., 2007 S. S. Fu, M. McFall, A. J. Saxon, J. C. Beckham, T. P. Carmody, and D. G. Baker et al., Post-traumatic stress disorder and smoking: A systematic review, Nicotine \& Tobacco Research 9 (11) (2007), pp. 1071-1084.

Galea et al., 2002 S. Galea, J. Ahern, H. Resnick, D. Kilpatrick, M. Bucuvalas, and J. Gold et al., Psychological sequelae of the September 11 terrorist attacks in New York City, The New England Journal of Medicine 346 (2002), pp. 982-987.

Gerrards-Hesse et al., 1994 A. Gerrards-Hesse, K. Spies, and F. W. Hesse, Experimental inductions of emotional states and their effectiveness: A review, British Journal of Psychology 85 (1994), pp. 55-78.

Haaga et al., 2004 D. A. F. Haaga, F. P. Thorndike, D. G. Friedman-Wheeler, M. Y. Pearlman, and R. A. Wernicke, Addictive Behaviors 29 (2004), pp. 1109-1122.

Heatherton et al., $1991-$ T. F. Heatherton, L. T. Kozlowski, R. C. Frecker, and K. O. Fagerstrom, The Fagerstrom Test for Nicotine Dependence: A revision of the Fagerstrom Tolerance Questionnaire, British Journal of Addiction 86 (1991), pp. 1119-1127.

Hufford, 2001 M. R. Hufford, An examination of mood effects on positive alcohol expectancies among undergraduate drinkers, Cognition and Emotion 15 (2001), pp. 593-613.

Kashdan et al., 2006 T. B. Kashdan, J. D. Elhai, and B. C. Frueh, Anhedonia and emotional numbing in combat veterans with PTSD, Behavior Research and Therapy 44 (3) (2006), pp. 457-467.

Kates and Mach, 2007 N. Kates and M. Mach, Chronic disease management for depression in primary care: A summary of the current literature and implications for practice, Canadian Journal of Psychiatry 52 (2007), pp. 77-85.

Khan et al., 2002 A. A. Khan, C. O. Gardner, C. A. Prescott, and K. S. Kendler, Gender differences in the symptoms of major depression in opposite-sex dizygotic twin pairs, American Journal of Psychiatry 159 (2002), pp. 1427-1429. 
Koenen et al., $2005 \rightarrow$ K. C. Koenen, B. Hitsman, M. J. Lyons, R. Niaura, J. McCaffery, and J. Goldberg et al., A twin registry study of the relationship between posttraumatic stress disorder and nicotine dependence in men, Archives of General Psychiatry 62 (2005), pp. 1258-1265.

Koenen et al., 2006 K. C. Koenen, B. Hitsman, M. J. Lyons, L. Stroud, R. Niaura, and J. McCaffery et al., Posttraumatic stress disorder and late-onset smoking in the Vietnam era twin registry, Journal of Consulting and Clinical Psychology 74 (2006), pp. 186-190.

Kramer et al., 2003 T. L. Kramer, B. M. Booth, X. Han, and D. K. Williams, Service utilization and outcomes in medically ill veterans with posttraumatic stress and depressive disorders, Journal of Traumatic Stress 16 (3) (2003), pp. 211-219.

Links and Comstock, $1990>$ R. W. Links and G. W. Comstock, Depressed mood and development of cancer, American Journal of Epidemiology 132 (1990), pp. 962-972.

Litt et al., 1990 M. D. Litt, N. L. Cooney, R. M. Kadden, and L. Gaupp, Reactivity to alcohol cues and induced moods in alcoholics, Addictive Behaviors 15 (1990), pp. 137-146.

Litz and Gray, 2002 B. T. Litz and M. J. Gray, Emotional numbing in posttraumatic stress disorder: Current and future research directions, Australian and New Zealand Journal of Psychiatry 36 (2002), pp. 198-204.

Maes et al., 2000 M. Maes, J. Mylle, L. Delmeire, and C. Altamura, Psychiatric morbidity and comorbidity following accidental man-made traumatic events: Incidence and risk factors, European Archives of Psychiatry and Clinical Neuroscience 250 (2000), pp. 156-162.

Marin, 1990 M. Marin, On the induction of mood, Clinical Psychology Review 10 (1990), pp. 669-697.

McChargue and Doran, 2009 D. E. McChargue and N. Doran, Cue-evoked positive affect, depression vulnerability and smoking years. American Journal of Health Behavior 33:1 (2009), pp. 49-57.

McChargue et al., 2004 D. E. McChargue, B. Spring, J. W. Cook, and C. Neumann, Reinforcement expectations explain the relationship between depressive history and smoking status in college students, Addictive Behaviors 29 (2004), pp. 991-994.

McClernon et al., 2005 F. J. McClernon, J. C. Beckham, S. L. Mozley, M. E. Feldman, S. R. Vrana, and J. E. Rose, The effects of trauma recall on smoking topography in posttraumatic stress disorder and non-posttraumatic stress disorder trauma survivors, Addictive Behaviors 30 (2005), pp. 247-257.

McNair et al., 1971 D. M. McNair, M. Lorr, and L. F. Droppelman, Manual for the Profile of Mood States, Educational and Industrial Testing Service, San Diego, CA (1971).

Nyenhuis et al., $1999>$ D. L. Nyenhuis, C. Yamamoto, T. Luchetta, A. Terrien, and A. Parmentier, Adult and geriatric normative data and validation of the Profile of Mood States, Journal of Clinical Psychology 55 (1999), pp. 79-86.

Oliver and Baumgart, 1985 J. M. Oliver and E. P. Baumgart, The Dysfunctional Attitude Scale: Psychometric properties and relation to depression in an unselected adult population, Cognitive Therapy and Research 9 (1985), pp. 161-167.

Otto et al., $2007 \longrightarrow$ M. W. Otto, B. A. Teachman, L. S. Cohen, C. N. Soares, A. F. Vitonis, and B. L. Harlow, Dysfunctional attitudes and episodes of major depression: Predictive validity and temporal stability in never-depressed, depressed, and recovered women, Journal of Abnormal Psychology 116 (2007), pp. 475-483.

Pfefferbaum et al., 2002 B. Pfefferbaum, S. S. Vinekar, R. P. Trautman, S. J. Lensgraf, C. Reddy, and N. Patel et al., The effect of loss and trauma on substance use behavior in individuals seeking support services after the 1995 Oklahoma City bombing, Annals of Clinical Psychiatry 14 (2002), pp. 89-95.

Rauch et al., 2006 S. A. M. Rauch, K. H. Morales, C. Zubritsky, K. Knott, and D. Oslin, Posttraumatic stress, depression, and health among older adults in primary care, American Journal of Geriatric Psychiatry 14 (4) (2006), pp. 316-324.

Segal et al., 1994 D. L. Segal, M. Hersen, and V. B. Van Hasselt, Reliability of the structured clinical interview for DSM-III-R: An evaluative review, Comprehensive Psychiatry 35 (1994), pp. 316-327.

Shalev et al., 1998 A. Y. Shalev, S. Freedman, T. Peri, D. Brandes, T. Sahar, and S. P. Orr et al., Prospective study of posttraumatic stress disorder and depression following trauma, American Journal of Psychiatry 155 (1998), pp. 630-637.

Smith et al., 2003 A. Smith, C. Brice, J. Nash, N. Rich, and D. J. Nutt, Caffeine and central noradrenaline: Effects on mood, cognitive performance, eye movements and cardiovascular function, Journal of Psychopharmacology 17 (3) (2003), pp. 282-292.

Spertus et al., 2003 I. L. Spertus, R. Yehuda, C. M. Wong, S. Halligan, and S. V. Seremetis, Childhood emotional abuse and neglect as predictors of psychological and physical symptoms in women presenting to a primary care practice, Child Abuse $\mathcal{E}$ Neglect 27 (2003), pp. 1247-1258.

Spring et al., 2007 B. Spring, J. W. Cook, B. Appelhans, A. Maloney, M. Richmond, and J. Vaughn et al., Nicotine effects on affective response in depression-prone smokers, Psychopharmacology 196 (2007), pp. 461-471.

Strong et al., 2004 D. R. Strong, R. A. Brown, C. W. Kahler, E. E. Lloyd-Richardson, and R. Niaura, Depression proneness in treatment-seeking smokers: A taxometric analysis, Personality and Individual Differences 36 (2004), pp. 1155-1170.

Tabachnick and Fidell, 2001 B. G. Tabachnick and L. S. Fidell, Computer-assisted research design and analysis, Allyn and Bacon, Boston (2001).

Tiffany and Drobes, $1990>$ S. T. Tiffany and D. J. Drobes, Imagery and smoking urges: The manipulation of affective content, Addictive Behaviors 6 (1990), pp. 531-539.

Tiffany and Hakenewerth, 1991 S. T. Tiffany and D. M. Hakenewerth, The production of smoking urges through an imagery manipulation: Psychophysiological and verbal manifestations, Addictive Behaviors 16 (1991), pp. 389-400.

Vaestfjaell, 2002 D. Vaestfjaell, Emotion induction through music: A review of the musical mood induction procedures, $M u-$ sicae Scientiae (2002), pp. 173-211 (special issue). 